\title{
Principles of Translational Control
}

\author{
John W.B. Hershey, ${ }^{1}$ Nahum Sonenberg, ${ }^{2}$ and Michael B. Mathews ${ }^{3}$ \\ ${ }^{1}$ Department of Biochemistry and Molecular Medicine, University of California, Davis, California 95616 \\ ${ }^{2}$ Goodman Cancer Research Centre and Department of Biochemistry, McGill University, Montreal, \\ Quebec H3A 1A3, Canada \\ ${ }^{3}$ Department of Medicine, Rutgers New Jersey Medical School, Newark, New Jersey 07103 \\ Correspondence: jwhershey@ucdavis.edu
}

Protein synthesis involves a complex machinery comprising numerous proteins and RNAs joined by noncovalent interactions. Its function is to link long chains of amino acids into proteins with precise sequences as encoded by the genome. Regulation of protein synthesis, called translational control, occurs both at a global level and at specific messenger RNAs (mRNAs). To understand how translation is regulated, knowledge of the molecular structures and kinetic interactions of its components is needed. This review focuses on the targets of translational control and the mechanisms employed.

$P^{r}$ roteins are vital constituents of all cells and are frequent products for secretion. Regulation of their synthesis contributes importantly to cell homeostasis, survival, differentiation, and many other functions. The process of protein synthesis is expensive, consuming much of a cell's energy. Consequently, it must be regulated to conserve energy. Equally important, cells need to make proteins in the right amounts, at the right time, and in the right place. To understand how cells deal with these challenges, an understanding of the mechanisms of protein synthesis and translational control is essential. Translational control is an important contributor to regulating gene expression and many disease states occur because of its dysfunction. This review examines the general principles of translational control.

\section{THE PROTEIN SYNTHESIS PATHWAY}

Protein synthesis involves the formation of peptide bonds that link the carboxyl group of one amino acid to the amino group of another to form a chain. Peptide bond formation is a relatively simple chemical process involving a nucleophilic attack by an amino group of one amino acid on the carboxyl group of another. Activation of the carboxyl group by the formation of an ester or anhydride facilitates the reaction, and requires energy. Such peptide bond formation reactions can then occur spontaneously. In theory, a simple enzyme should be capable of catalyzing peptide bond formation. However, the synthesis of proteins in cells involves a very complex machinery comprising ribosomes, numerous RNAs and proteins, and,

Editors: Michael B. Mathews, Nahum Sonenberg, and John W.B. Hershey

Additional Perspectives on Translation Mechanisms and Control available at www.cshperspectives.org

Copyright (C) 2019 Cold Spring Harbor Laboratory Press; all rights reserved; doi: 10.1101/cshperspect.a032607

Cite this article as Cold Spring Harb Perspect Biol 2019;11:a032607 
J.W.B. Hershey et al.

of course, amino acids and energy. Such complexity is needed because the attachment of amino acids must occur in such a way that the resulting sequence of amino acids in the protein is generated with high fidelity according to the genetic code. This sequence information comes from the messenger RNA (mRNA), which helps select the next amino acid and aligns it with the nascent peptide chain.

A growing peptide can, in theory, add the next amino acid either to its amino terminus or its carboxyl terminus. Chemists developed a nonphysiological method called solid-phase protein synthesis that involves attachment of the carboxyl group of the first amino acid to a solid support, followed by the sequential addition of activated amino acids, leading to elongation at the amino terminus of the nascent peptide chain (Merrifield 1964). In contrast, cells synthesize proteins by first generating an activated amino acid attached to a specific transfer RNA (tRNA) through an ester bond. A specific activated amino acid (aa-tRNA) is selected through interaction between a codon in the mRNA and the anticodon in its tRNA. Bound to an adjacent upstream codon in the mRNA is the nascent peptide, attached to its tRNA through its carboxy terminal ester bond. This activated nascent peptide (in the peptidyltRNA) is transferred to the amino group of the adjacent aa-tRNA on the mRNA (for details of this mechanism, see Dever et al. 2018; Rodnina 2018). Thus, native protein synthesis involves elongation at the carboxyl terminus of the nascent peptide. In contrast to an amino-terminal extension mechanism, carboxyl-terminal extension involves the binding of the peptidyl-tRNA and incoming aa-tRNA to adjacent codons in the mRNA. Therefore, each reaction in the multiple cycles of elongation occurs similarly at the same place on the ribosome, enabling protein synthesis to occur with high fidelity. A brief description of the process of protein synthesis follows.

The translation pathway in cells can be broken into four stages: initiation, elongation, termination, and ribosome recycling. In eukaryotes, the major eukaryotic cellular translation components are two ribosomal subunits, 40S and 60S, composed of four RNAs and over 80 proteins; an mRNA; aminoacyl-tRNAs (aatRNAs) formed by attaching each of the 20 amino acids to its specific tRNAs through an energy-consuming reaction catalyzed by their specific aminoacyl-tRNA synthetases; protein factors that interact with the other components; and GTP and ATP, providing energy through their hydrolysis.

Protein synthesis on most mRNAs begins by forming a complex containing the $40 \mathrm{~S}$ small ribosomal subunit and the initiator methionyltRNA $_{\mathrm{i}}\left(\right.$ Met-tRNA $\left._{\mathrm{i}}\right)$, which then binds to the $5^{\prime}$ end of the mRNA. The resulting complex, called the preinitiation complex (PIC), also contains many protein factors (initiation factors) as well as GTP and ATP. The PIC then scans down the mRNA through an energy-driven process until the anticodon in the Met-tRNA $\mathrm{A}_{\mathrm{i}}$ base-pairs with the initiator codon (usually AUG) in the mRNA. This interaction causes termination of PIC scanning, the hydrolysis of bound GTP, and ejection of many of the initiation factors, enabling junction with the $60 \mathrm{~S}$ ribosomal subunit to form an $80 \mathrm{~S}$ initiation complex competent to enter the elongation phase of protein synthesis. An important feature of the initiation pathway is that no covalent bond is made or broken, other than those involved in the charging of methionine to its tRNA and the hydrolysis of GTP and ATP. Thus, formation of an 80 S initiation complex mainly involves noncovalent interactions between the numerous proteins and RNAs. A detailed description of the eukaryotic initiation pathway is found in Merrick and Pavitt (2018).

Following initiation complex formation, with the Met-tRNA $A_{i}$ bound to the $\mathrm{P}$ (peptidyl) site of the 80S ribosome, a new aa-tRNA is selected and binds into the ribosomal A (aminoacyl) site through base-pairing between its anticodon and the codon in the mRNA just downstream of the initiator AUG. The ribosome catalyzes the transfer of the methionyl group from its tRNA to the amino group of the aatRNA in the A site, resulting in the formation of the first peptide bond. The peptidyl-tRNA in the A site, together with the mRNA, move along the ribosome into the $\mathrm{P}$ site (called translocation). Then the mRNA codon in the A site 
binds a new aa-tRNA, again by base-pairing. The repetitive aa-tRNA binding, peptide bond formation, and translocation steps, called the elongation phase of protein synthesis, are promoted by proteins called elongation factors that transiently associate with the components involved. Besides breaking the aa-tRNA ester bond to form the peptide bond and the hydrolysis of GTP, elongation consists of numerous noncovalent interactions that enable the alignments on the ribosome to produce a proper amino acid sequence in the growing peptide chain. This pathway is described in detail in Dever et al. (2018).

When the ribosome encounters an A-site mRNA codon of UAA, UGA, or UAG, no aatRNA recognizes it. Instead, a protein release factor binds into the A site and catalyzes the hydrolysis of the nascent peptidyl-tRNA in the $\mathrm{P}$ site, resulting in termination of protein synthesis. Additional proteins then interact with the ribosome to promote the ejection of the tRNA and mRNA and dissociation of the ribosome into its subunits (a process called ribosome recycling). Termination and recycling are described in detail in Hellen (2018).

Most of the principal features of the protein synthesis pathway are common to all domains of life, especially the structure of the ribosome responsible for aa-tRNA binding to mRNA codons and peptide bond formation. The components and mechanism of initiation may differ significantly, however. For example, the scanning mechanism for start-site selection in eukaryotes is replaced in prokaryotes by a mechanism involving base-pairing between ribosomal RNA and mRNA (reviewed by Rodnina 2018). Alternative initiation mechanisms are also exploited in some eukaryotic systems (see Kwan and Thompson 2018) and their viruses (SternGinossar et al. 2018), often for regulation.

\section{FEATURES OF TRANSLATIONAL CONTROL}

The cellular level of a protein is determined by a balance between its rates of synthesis and degradation. The synthesis of a specific protein requires transcription of its mRNA, a process that is highly regulated (transcriptional control).
mRNAs may also undergo several critical modifications at their termini (capping and polyadenylation), as well as internally (splicing, methylation, etc.). In addition to transcription, mRNA stability also affects mRNA levels. But mRNA levels alone do not determine the amounts of proteins in cells, as some mRNAs are not actively translated, or are translated poorly, being bound to proteins, called messenger ribonucleoproteins (mRNPs), or sequestered in stress granules (SGs) or processing bodies (PBs) (Ivanov et al. 2018). In addition, translation rates are not always uniform among different mRNA species or along individual mRNAs (Biswas et al. 2018; Ingolia et al. 2018).

The synthesis rate of a specific protein is determined by the number of ribosomes translating its mRNA together with the overall rate of peptide bond formation. The number of translating ribosomes is determined by the number of active mRNAs, the coding length of the mRNA, the rate of ribosome attachment to the mRNA (initiation), and rate of elongation. The average rate of elongation in eukaryotic cells is normally quite fast (often about five amino acids per second), whereas the initiation step is usually ratelimiting (around one every 5 seconds for strongly translated mRNAs) (Palmiter 1975). However, if elongation rates are greatly reduced, this will affect initiation rates as well, as the ribosome must move out of the initiation region of the mRNA to enable the next ribosome to initiate. A ribosome bound at the initiator codon must move downstream at least five codons ( $\sim 1$ second when fast) before a new initiating ribosome can bind there.

How are the rates of global protein synthesis measured? A classical method is to measure the rate of radioactive amino acid incorporation into proteins. This can be applied to measuring both global rates and specific protein rates, the latter generally involving immunoprecipitation. Stable isotope labeling with amino acids in cell culture (SILAC) enables quantification of newly synthesized proteins by mass spectrometry (Schwanhausser et al. 2011). A third method is to determine the number of active ribosomes and their elongation/termination rate. The elongation/termination rate is equal to the average 
J.W.B. Hershey et al.

size of synthesized proteins divided by the ribosome transit time, measured by radioactive amino acid labeling followed by centrifugation at different times to separate released proteins from ribosome-bound nascent proteins (Fan and Penman 1970). To determine the rate of initiation for a protein, measuring its polysome size divided by the ribosome transit time can be employed (Palmiter 1975). Polysome profiling, a method involving separation of polysome sizes by sucrose gradient centrifugation, is used to detect changes in initiation rates, as the rate of initiation greatly affects polysome size. However, elongation rates also affect polysome size, so measurement of the elongation rate also is needed. Ribosome profiling (Ingolia et al. 2018), which entails a global analysis of ribosome-protected mRNA segments, also provides information about global and specific protein synthesis rates, as well as fine details of ribosomal progression along an mRNA, and is now frequently employed in studying translational control.

Most mechanisms of translational control in cells affect the rate of initiation. By regulating initiation, rates of protein synthesis can be quickly altered and can affect specific mRNAs differently. The rates of peptide bond formation (elongation) and termination/recycling also can affect the rate of ribosome attachment. If all nascent peptides are completed as full-length protein products, then the initiation rate defines the overall protein synthesis rate during steadystate protein synthesis. Because both short and long mRNA open reading frames (ORFs) may initiate at similar rates, resulting, respectively, in light versus heavy polysomes, both large and small proteins can, in principle, be made at similar rates.

What determines the initiation rate of an mRNA, and how is this regulated? Specific mRNAs can initiate with different efficiencies because of their sequences or secondary structures that affect interactions with the translational machinery. Trans-acting factors (proteins, small RNAs, riboswitches/ligands) that bind specific mRNAs can enhance or inhibit the recruitment of ribosomes (see Breaker 2018; Duchaine and Fabian 2018). The cellular levels of available ribosomes, initiation factors, and
Met-tRNA $A_{i}$ influence initiation rates. The initiation factors also can be modified posttranslationally, for example, by phosphorylation (see Proud 2018; Wek 2018), and the extent of such modifications can affect factor activities and initiation rates.

Protein synthesis is one of the most energyconsuming pathways in cell metabolism (Rolfe and Brown 1997); two high-energy phosphate bonds are used to activate the amino acid by producing aa-tRNA and two to generate the peptide bond (elongation), making four per amino acid incorporated. Although six highenergy phosphate bonds are broken to synthesize one mRNA codon, the codon is translated multiple times, making protein synthesis much more expensive than mRNA transcription. To regulate the translation rate by slowing elongation and/or termination is expensive, as the translating ribosome is less productive. Such slower rates would require more ribosomes to generate the same amounts of protein, and ribosomes are large and very expensive to make. However, inhibition of elongation can rapidly decrease protein synthesis and thereby conserve energy when the cell's energy level drops suddenly (Wek 2018). Modest reductions in elongation rates (e.g., by phosphorylation of elongation factors; Dever et al. 2018; Proud 2018) or rare codon usage, are sometimes used to adjust translation rates to coordinate cotranslation protein secretion and folding. Thus, the rate of global or specific protein synthesis can be determined by the sum of all of these possible regulatory mechanisms. If a number of different mechanisms affect the same mRNA at the same time, the individual effect of each mechanism could be small, difficult to detect, yet nevertheless important.

\section{REGULATORY MECHANISMS}

The targets for regulation at initiation are very numerous, as it is not a covalent bond reaction that is being regulated, but rather the many noncovalent interactions required to form initiation complexes in the correct conformation. An important target for the regulation of global translation is eukaryotic initiation factor (eIF)2, 
whose phosphorylation inhibits its recycling by eIF2B and thus its ability to bind Met-tRNA $A_{i}$, an interaction involved in the translation of nearly all mRNAs (Merrick and Pavitt 2018; Wek 2018). Another target is eIF4E, the cap-binding protein, which is involved in selecting an mRNA and also promotes the scanning process through stimulation of the RNA helicase activity of eIF4A. Its down-regulation by binding eIF4Ebinding proteins (4E-BPs) (Merrick and Pavitt 2018; Proud 2018; Robichaud et al. 2018) can affect mRNAs differently, as some mRNAs require high eIF4E activity, whereas others do not. Many other targets of initiation are described in the scientific literature. Elongation rates also can be affected by codon usage, tRNA levels, and elongation factor modifications (Dever et al. 2018; Proud 2018).

\section{Posttranslational Modifications of Translational Machinery Proteins}

The most common method for regulating protein synthesis is through phosphorylation of the proteins involved. Phosphorylation levels are controlled by protein kinases and phosphatases whose activities are responsive to conditions in the cell. As mentioned above, the most important targets are eIF2 and the 4E-BPs (Merrick and Pavitt 2018). In addition, other initiation and elongation factors are phosphorylated and contribute to translational control (Proud 2018; Wek 2018). To rigorously establish a correlation between phosphorylation and translational control, it is important to identify the phosphorylation site and measure the extent of phosphorylation. To establish that a phosphorylation actually causes a change in translational efficiency, mutant forms of the target protein that mimic or prevent phosphorylation are frequently tested, as are inhibition or knockout of specific protein kinases.

\section{mRNA Structure and Complexes}

mRNAs have unique sequences, both in their coding and noncoding regions. The lengths and sequences of the $5^{\prime}$ and $3^{\prime}$ untranslated regions (UTRs) differ and can affect interactions with the translational machinery. Alternate promoters and alternative splicing can generate different mRNAs that encode the same protein, but their translational efficiencies may not be identical. Cellular mRNAs can be modified (Peer et al 2018) and can bind various proteins to form mRNPs (Mitchell and Parker 2014) that may differ in their abilities to initiate translation. mRNAs also interact with small RNAs (microRNAs) that affect initiation (Duchaine and Fabian 2018). A current challenge is to determine the precise structure of an mRNA, especially as found in vivo when bound with cellular proteins.

\section{mRNA Levels}

mRNA levels obviously play a role in determining the amounts of protein produced by the translational machinery. As mentioned earlier, mRNA levels are determined by their rates of transcription and processing, but also by their rates of transport into the cytoplasm (Biswas et al. 2018) and by their rates of degradation that may be coupled to or influenced by their translation (Heck and Wilusz 2018), by their sequences (Karousis and Mühlemann 2018), as well as by microRNAs (Duchaine and Fabian 2018). mRNAs also can be sequestered in PBs and SGs (Ivanov et al. 2018) where they are not being translated. Finally, mRNAs may bind other proteins to form mRNPs, which undergo either enhanced or reduced translation. Because stimulating eukaryotic gene expression through transcriptional control requires a considerable amount of time to elevate mRNA levels, and in some cases to transport them to specific cellular regions (e.g., in neurons), translational control can be much more rapid.

\section{FUTURE PROSPECTS}

Although much is known about the mechanism of protein synthesis and its control, there are aspects of our understanding that need to be improved. The structure of ribosomes and protein factors provide much needed information (Jobe et al. 2018), but the structures of initiation complexes remain elusive. What is the actual structure of a native mRNA, especially 
J.W.B. Hershey et al.

its $5^{\prime} \mathrm{UTR}$, and how is it altered by the binding of proteins or small RNAs? Another area concerns the kinetics of the various noncovalent interactions involved in initiation (Prabhakar et al. 2018; Sokabe and Fraser 2018). What is the order of binding of the various components? What are their rates of binding and dissociation, and how are these rates affected by their levels, modifications, and order of binding? How is a given mRNA selected from the pool of mRNAs in the cytoplasm, as this is one of the most important reactions for translational control? A thorough knowledge of the mechanisms and rates of protein synthesis is needed to better understand how translational control contributes to the regulation of gene expression.

\section{REFERENCES}

${ }^{*}$ Reference is also in this collection.

* Biswas J, Liu Y, Singer RH, Wu B. 2018. Fluorescence imaging methods to investigate translation in single cells. Cold Spring Harb Perspect Biol doi: 10.1101/cshperspect. a032722.

* Breaker RR. 2018. Riboswitches and translation control. Cold Spring Harb Perspect Biol doi: 10.1101/cshperspect. a032797.

* Dever TE, Dinman JD, Green R. 2018. Translation elongation and recoding in eukaryotes. Cold Spring Harb Perspect Biol doi: 10.1101/cshperspect.a032649.

* Duchaine TF, Fabian MR. 2018. Mechanistic insights into microRNA-mediated gene silencing. Cold Spring Harb Perspect Biol doi: 10.1101/cshperspect.a032771.

Fan H, Penman S. 1970. Regulation of protein synthesis in mammalian cells. II: Inhibition of protein synthesis at the level of elongation. Proc Natl Acad Sci 87: 328-332.

* Heck AM, Wilusz J. 2018. The interplay between the RNA decay and translation machinery in eukaryotes. Cold Spring Harb Perspect Biol doi: 10.1101/cshperspect. a032839.

* Hellen CUT. 2018. Translation termination and ribosome recycling in eukaryotes. Cold Spring Harb Perspect Biol doi: 10.1101/cshperspect.a032656.

* Ingolia NT, Hussmann JA, Weissman JS. 2018. Ribosome profiling: Global views of translation. Cold Spring Harb Perspect Biol doi: 10.1101/cshperspect.a032698.

* Ivanov P, Kedersha N, Anderson P. 2018. Stress granules and processing bodies in translational control. Cold Spring Harb Perspect Biol doi: 10.1101/cshperspect.a032813.
* Jobe A, Liu Z, Gutierrez-Vargas C, Frank J. 2018. New insights into ribosome structure and function. Cold Spring Harb Perspect Biol doi: 10.1101/cshperspect. a032615.

* Karousis ED, Mühlemann O. 2018. Nonsense-mediated mRNA decay begins where translation ends. Cold Spring Harb Perspect Biol doi: 10.1101/cshperspect.a032862.

* Kwan T, Thompson SR. 2018. Noncanonical translation initiation in eukaryotes. Cold Spring Harb Perspect Biol doi: 10.1101/cshperspect.a032672.

* Merrick WC, Pavitt GD. 2018. Protein synthesis initiation in eukaryotic cells. Cold Spring Harb Perspect Biol doi: 10.1101/cshperspect.a033092.

Merrifield RB. 1964. Solid-phase peptide synthesis. 3: An improved synthesis of bradykinin. Biochemistry 3: 13851390.

Mitchell SF, Parker R. 2014. Principles and properties of eukaryotic mRNPs. Mol Cell 54: 547-558.

Palmiter R. 1975. Quantitation of parameters that determine the rate of ovalbumin synthesis. Cell 4: 189-197.

* Peer E, Moshitch-Moshkovitz S, Rechavi G, Dominissini D. 2018. The epitranscriptome in translation regulation. Cold Spring Harb Perspect Biol doi: 10.1101/cshperspect. a032623.

* Prabhakar A, Puglisi EV, Puglisi JD. 2018. Single-molecule florescence applied to translation. Cold Spring Harb Perspect Biol doi: 10.1101/cshperspect.a032714.

* Proud CG. 2018. Phosphorylation and signal transduction pathways in translational control. Cold Spring Harb Perspect Biol doi: 10.1101/cshperspect.a033050.

* Robichaud N, Sonenberg N, Ruggero D, Schneider RJ. 2018. Translational control in cancer. Cold Spring Harb Perspect Biol doi: 10.1101/cshperspect.a032896.

* Rodnina MV. 2018. Translation in prokaryotes. Cold Spring Harb Perspect Biol doi: 10.1101/cshperspect.a032664.

Rolfe DF, Brown GC. 1997. Cellular energy utilization and molecular origin of standard metabolic rate in mammals. Physiol Rev 77: 731-758.

Schwanhausser B, Busse D, Li N, Dittmar G, Schuchhardt J, Wolf J, Chen W, Selbach M. 2011. Global quantification of mammalian gene expression control. Nature 473: 337 342 .

* Sokabe M, Fraser CS. 2018. Toward a kinetic understanding of eukaryotic translation. Cold Spring Harb Perspect Biol doi: 10.1101/cshperspect.a032706.

* Stern-Ginossar N, Thompson SR, Mathews MB, Mohr I. 2018. Translational control in virus-infected cells. Cold Spring Harb Perspect Biol doi: 10.1101/cshperspect. a033001.

* Wek RC. 2018. Role of eIF $2 \alpha$ kinases in translational control and adaptation to cellular stresses. Cold Spring Harb Perspect Biol doi: 10.1101/cshperspect.a032870. 


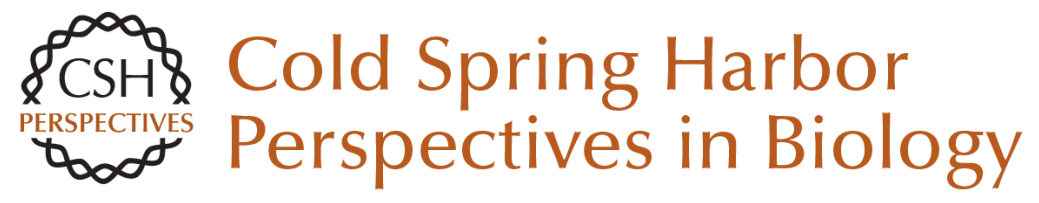

\section{Principles of Translational Control}

John W.B. Hershey, Nahum Sonenberg and Michael B. Mathews

Cold Spring Harb Perspect Biol 2019; doi: 10.1101/cshperspect.a032607 originally published online June 29, 2018

\section{Subject Collection Translation Mechanisms and Control}

Protein Synthesis and Translational Control: A Historical Perspective

Soroush Tahmasebi, Nahum Sonenberg, John W.B. Hershey, et al.

Translational Control in the Brain in Health and Disease

Wayne S. Sossin and Mauro Costa-Mattioli

Phosphorylation and Signal Transduction

Pathways in Translational Control Christopher G. Proud

Translational Control during Developmental Transitions

$$
\text { Felipe Karam Teixeira and Ruth Lehmann }
$$

Stress Granules and Processing Bodies in Translational Control

Pavel Ivanov, Nancy Kedersha and Paul Anderson

Fluorescence Imaging Methods to Investigate

Translation in Single Cells

Jeetayu Biswas, Yang Liu, Robert H. Singer, et al.

Translational Control in Virus-Infected Cells Noam Stern-Ginossar, Sunnie R. Thompson, Michael B. Mathews, et al.

Nonsense-Mediated mRNA Decay Begins Where Translation Ends

Evangelos D. Karousis and Oliver Mühlemann
Principles of Translational Control John W.B. Hershey, Nahum Sonenberg and Michael B. Mathews

The Epitranscriptome in Translation Regulation Eyal Peer, Sharon Moshitch-Moshkovitz, Gideon Rechavi, et al.

Translational Control in Cancer Nathaniel Robichaud, Nahum Sonenberg, Davide Ruggero, et al.

Roles of Long Noncoding RNAs and Circular

RNAs in Translation Marina Chekulaeva and Nikolaus Rajewsky

Ribosome Profiling: Global Views of Translation Nicholas T. Ingolia, Jeffrey A. Hussmann and Jonathan S. Weissman

Noncanonical Translation Initiation in Eukaryotes Thaddaeus Kwan and Sunnie R. Thompson

Mechanistic Insights into MicroRNA-Mediated Gene Silencing Thomas F. Duchaine and Marc R. Fabian

Toward a Kinetic Understanding of Eukaryotic Translation Masaaki Sokabe and Christopher S. Fraser

For additional articles in this collection, see http://cshperspectives.cshlp.org/cgi/collection/

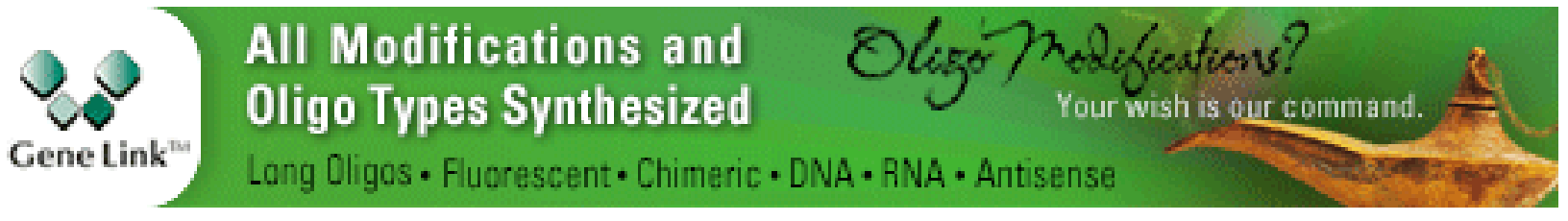

